This document is the accepted manuscript version of the following article:

Ramasubramanian, L., Kákay, A., Fowley, C., Yildirim, 0., Matthes, P., Sorokin, S., Titova, A., Hilliard, D., Böttger, R., Hübner, R., Gemming, S., Schulz, S. E., Kronast, F., Makarov, D., Fassbender, J., \& Deac, A. (2020). Tunable magnetic vortex dynamics in ion-implanted permalloy disks. ACS Applied Materials and Interfaces, 12(24), 27812-27818. https://doi .org/10.1021/acsami .0c08024

\title{
Tunable magnetic vortex dynamics in ion-implanted permalloy disks
}

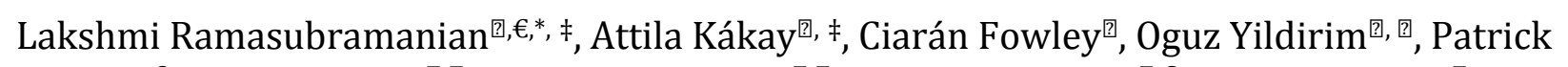

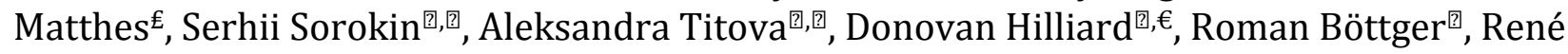
Hübner $^{\natural}$, Sibylle Gemming ${ }^{\natural, €}$, Stefan E. Schulz ${ }^{€, €}$, Florian Kronast ${ }^{\$}$, Denys Makarov ${ }^{\natural}$, Jürgen Fassbender ${ }^{\text {巴, }}$ and Alina Deac ${ }^{\text {国 }}$.

${ }^{\square}$ Helmholtz-Zentrum Dresden-Rossendorf, Institute of Ion Beam Physics and Materials Research, 01328, Dresden, Germany

${ }^{€}$ Technische Universität Chemnitz, 09126, Chemnitz, Germany

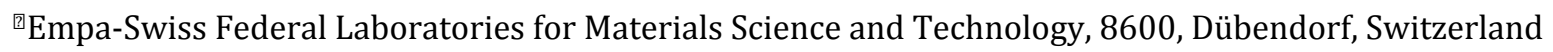

${ }^{£}$ Fraunhofer Institute for Electronic Nano Systems, 09126, Chemnitz, Germany

${ }^{\square}$ Institute of Solid State Physics, TU Dresden, 01069, Dresden, Germany

${ }^{\$}$ Helmholtz-Zentrum Berlin für Materialen und Energie, 12489 Berlin, Germany

KEYWORDS: electrical detection, vortex dynamics, frequency tunability, ion implantation, reduced saturation magnetization

\begin{abstract}
Nanoscale, low-phase noise, tunable transmitter-receiver links are key for enabling the progress of wireless communication. We demonstrate that vortex-based spin-torque nano-oscillators, which are intrinsically low-noise devices due to their topologically-protected magnetic structure, can achieve frequency tunability when submitted to local ion implantation. In the experiments presented here, the gyrotropic mode is excited with spin-polarized alternating currents and anisotropic magnetoresistance measurements yield discreet frequencies from a single device. Indeed, chromium-implanted regions of permalloy disks exhibit different saturation magnetisation than neighbouring, non-irradiated areas, and thus different resonance frequency, corresponding to the specific area where the core is gyrating. Our study proves that such devices can be fabricated without the need of further lithographical steps, suggesting ion irradiation can be a viable and costeffective fabrication method for densely-packed networks of oscillators.
\end{abstract}

Introduction: Using spin-polarized currents to manipulate the magnetic state of nano-objects has proven an effective method for designing new spintronic devices for a variety of applications. Indeed, current-induced magnetization switching is exploited as writing method for non-volatile magnetic random access memories (MRAMs), which are currently being commercialized as embedded cache memories, as a replacement for SRAM devices, but potentially also as a persistent DRAM alternative. On the other hand, spin-transfer-driven steady-state precession has been proposed as functioning principle for radiofrequency (RF) oscillators for wireless communication applications. Indeed, it has been shown that devices based on single-domain magnetic tunnel junctions can emit signals with output powers of several $\mu \mathrm{W}$ at frequencies of the order of $\mathrm{GHz}$ or tens of $\mathrm{GHz}$; moreover, their frequency can be tuned by changing the applied current ${ }^{1}$. The main drawbacks of uniformly magnetized nanopillars as RF oscillators for wireless communication remain their poor quality factors as consequence of inhomogeneous broadening of the output signal, and the large $1 / \mathrm{f}$ noise. Both issues are caused by incoherent magnetization dynamics ${ }^{2}$, though more elaborate device designs have demonstrated considerable improvement ${ }^{3}$. Vortex-based spin-torque oscillators, on the other hand, offer narrow linewidth and are less susceptible to thermal noise, but are, unfortunately, single-frequency devices ${ }^{4-6}$. Indeed, the magnetic "vortex" is a potential candidate for future spintronic devices, such as low-noise magnetic sensors ${ }^{7}$, frequency sensors ${ }^{8,9}$, and tunable magnonic crystals ${ }^{10}$. More recently, coupled vortex spin-torque oscillators have been integrated into complex circuits which can be 'trained' to recognize a series of sounds ${ }^{11}$ by exploiting their ability to synchronize on external ac field or current signals, offering an exciting new path for neuro-inspired computing ${ }^{12-16}$ by mimicking the dynamical nature of the brain. Commercial applications of magnetic vortices require tuning of the output frequency by external parameters, such as applied fields or spin-polarized currents. Indeed, spin-torque oscillators have been shown to exhibit output powers and quality factors compatible with wireless communication ${ }^{1}$. However, the frequency tunability of vortex-based devices is limited, since the gyrotropic frequency is specific to the 
individual sample design. To overcome this drawback, in this work, regions of
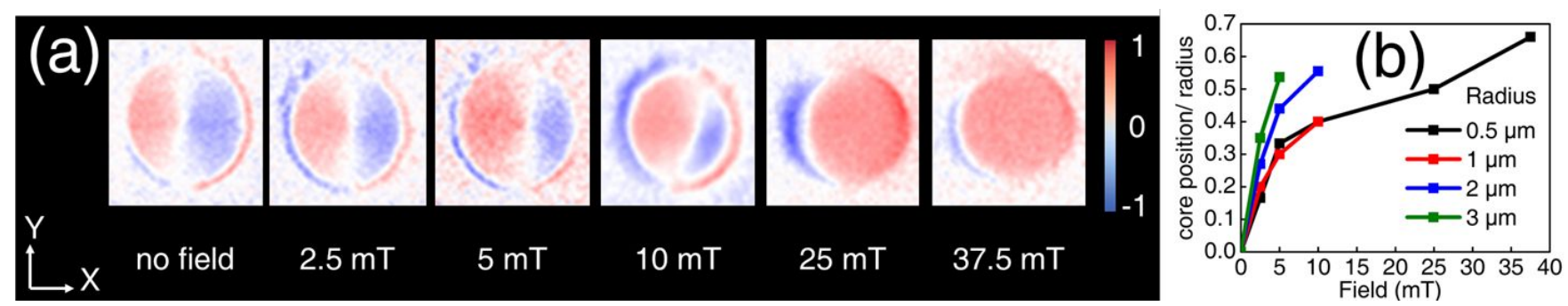

Figure 1: (a) XMCD images of a non-irradiated $30 \mathrm{~nm}$ thick Py disk with $1 \mu \mathrm{m}$ radius at different magnetic fields applied in-plane. The images show the colour-coded in-plane magnetisation direction when the field is applied in the Y direction. (b) Core displacement from the center, normalized to the disk radius, as a function of magnetic field, for $30 \mathrm{~nm}$ thick Py disks, with $0.5 \mu \mathrm{m}$, $1 \mu \mathrm{m}, 2 \mu \mathrm{m}$ and $3 \mu \mathrm{m}$ radius, as derived from the XMCD experiment.

different saturation magnetisations are induced in single magnetic disks by ion implantation. Implanted regions yield different resonance frequencies corresponding to the specific area where the core is precessing. We demonstrate the realisation of the multiple resonance frequencies in a single magnetic disk experimentally and support our results with micromagnetic simulations.

The vortex state in a ferromagnetic disk is a naturally forming flux closure state, where the in-plane magnetisation circulates around an out-of-plane magnetised core region. The out-of-plane magnetisation at the center of such a flux closure structure is a result of the exchange interaction. This overpowers the magnetostatic interaction, thereby preventing a singularity of the exchange energy density and forming an extremely stable structure $^{17}$. The magnetisation direction of the vortex core, known as the polarity, can point either upwards or downwards and thus can be considered as the ' 0 ' and ' 1 ' state of a storage element, i.e., a 'bit' of information. The inplane flux closure direction ${ }^{18}$ can also be used as an information carrier by exploiting the clockwise or counterclockwise winding direction ${ }^{19}$. Depending on the respective combinations of in-plane circulation and core polarity, four different configurations of vortex states can exist. Thus, a magnetic vortex can simultaneously store two bits of information and can be exploited for the realisation of data storage devices.

Despite being a highly stable structure in the absence of magnetic fields, the position of the vortex core can be altered by applying in-plane magnetic fields. When an external magnetic field is applied to the vortex in equilibrium, the vortex is displaced, distorting the in-plane circular arrangement of the local magnetisation. This results in an internal demagnetising field opposing the displacement of the vortex. Once the external field $\boldsymbol{H}$ is removed, the vortex core moves along a spiral trajectory and gyrates around the equilibrium position, before eventually settling down back to its equilibrium state. The radius of the precession trajectory changes due to damping. This periodic circular motion can be maintained by using an oscillating field or current which balances the damping, tuned to the resonant frequency of the sample. The vortex gyration at its resonant frequency can be electrically detected by using the anisotropic magnetoresistance (AMR), as initially shown by S. Kasai et $a^{20}$. From an application point of view, magnetisation dynamics driven by electrical (spin-polarized) currents is preferable to magnetic fields, as it provides perfect selectivity due to spatial localization.

The natural resonance of a vortex is a gyrotropic motion of the vortex core. Treating the vortex as a particle in a potential, its motion can be described by the Thiele equation $^{21}$, which expresses the relation between the velocity of the vortex core, the gyrovector and the effective magnetostatic potential in which the vortex core is moving. Solving this equation yields a gyroscopic frequency which is proportional to the spatial derivative of the effective magnetostatic potential at the radius of the trajectory and inversely proportional to the gyrovector ${ }^{21}$ :

$$
f=\frac{1 d W}{2 \pi r|G| d r}(r)
$$

where $r$ is the trajectory radius of the circular core motion and $G$ is the gyrovector. For an isolated disk with homogeneous magnetization, the potential $W$ adopts the symmetry of the circular nanomagnet and thus exhibits a parabola-like shape with its minimum located at the disk center, allowing for equation 1 to be simplified as follows ${ }^{22}$ :

$$
f_{0} \propto \mu_{0} M_{\mathrm{s}} \frac{h}{R}
$$

This dependence on the geometrical properties and material properties poses an obstacle for realizing frequency tunability over a large range in a single spintorque-driven vortex oscillator, as only one frequency is possible for a given combination of $h, R$ and $M_{\mathrm{s}}$. In order for a single device to be able to exhibit more than one frequency, two scenarios are possible: either spatially altering the topography ${ }^{23}$, or spatially varying the saturation magnetization. In this work, we show experimentally that by varying the $M_{\mathrm{s}}$ of single disks using local ion beam irradiation, multiple gyrotropic frequencies can be obtained in single devices, thus allowing for frequency tunability. The experimental results are qualitatively supported by micromagnetic simulations.

Results: Permalloy (Py- $\left.\mathrm{Ni}_{80} \mathrm{Fe}_{20}\right)$ disks with different radii were prepared by means of electron beam lithography followed by conventional lift-off technique, in order to experimentally confirm that a disk with concentric regions of different saturation magnetization can exhibit multiple, discrete frequencies, as predicted by micromagnetic simulations. The saturation magnetization was tuned in the different regions of the disks by using ion-beam irradiation. The spontaneously forming vortex states in the nonirradiated disks with different radii were confirmed with X- 
ray magnetic circular dichroism using photo-emission electron microscopy (XMCD-PEEM) measurements. Details on the sample fabrication and measurements techniques can be found in the methods section.

XMCD images of a $1 \mu \mathrm{m}$ radius, $30 \mathrm{~nm}$ thick Py disk measured for different applied magnetic fields are shown, as a representative example, in Figure 1(a). Red and blue contrasts refer to magnetisation vectors with the same amplitude but opposite direction. The absence of a net contrast is indicated by white. The ring-like contrast observed around the disks is an artifact of drift corrections in the measurement. As seen from Figure 1(a), in this $1 \mu \mathrm{m}$ radius disk, the vortex annihilates at a magnetic field between $10 \mathrm{mT}$ and $25 \mathrm{mT}$.

The results of measurements performed on $30 \mathrm{~nm}$ thick Py disks with different radii, ranging from $0.5 \mu \mathrm{m}$ to $3 \mu \mathrm{m}$, are plotted in Figure 1(b), showing the normalized core positions (with respect to the radius) as a function of the applied magnetic field. Similar to the example shown in Figure 1(a), in all cases, when the field is increased, the vortex core moves away from the center and is eventually expelled, resulting in a homogeneously magnetised, single domain disk.

Smaller external fields are required to expel the vortex core from bigger disks when compared to smaller disks: for instance, the vortex core from a $3 \mu \mathrm{m}$ radius disk annihilates between $5 \mathrm{mT}$ and $10 \mathrm{mT}$, whereas a $0.5 \mu \mathrm{m}$ radius disk requires more than $35 \mathrm{mT}$ in-plane magnetic field before reaching the single domain state. This strong dependence of the annihilation field on the disk radius can be explained by the increasing contribution of the magnetostatic energy with decreasing radius, making vortices in smaller disks more stable ${ }^{24}$. Note that the absolute core displacement versus field is much larger for larger disks, since Figure 1(b) shows the core position normalized to the disk radius.

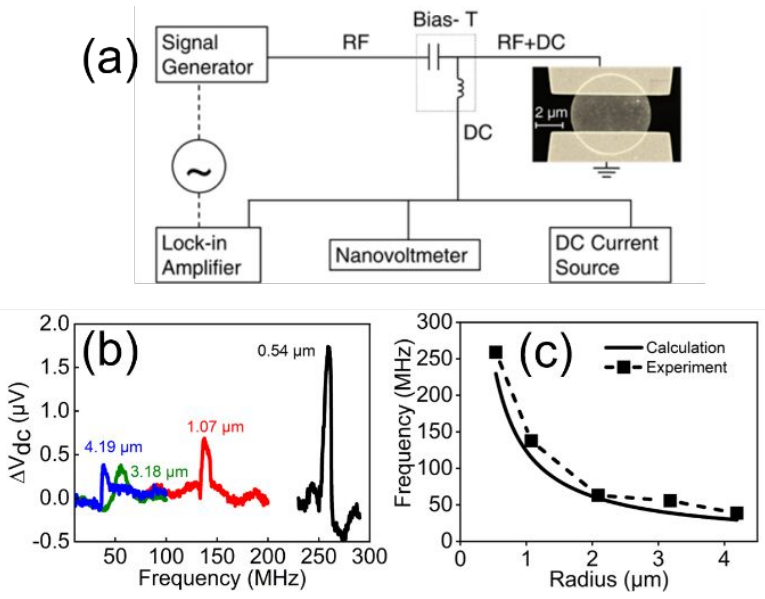

Figure 2: (a) Schematic of the measurement technique for dynamic measurements. (b) Electrically detected dynamics of vortex structures for non-irradiated disks with $25 \mathrm{~nm}$ Py for different disk radii, measured at current density (j), magnetic field) of $(\mathrm{j}, H)=\left(0.09 \times 10^{11} \mathrm{~A} / \mathrm{m}^{2}, 0.1 \mathrm{mT}\right)$ for a $4.19 \mu \mathrm{m}$ radius disk, $(\mathrm{j}, H)=\left(0.28 \times 10^{11} \mathrm{~A} / \mathrm{m}^{2}, 0.5 \mathrm{mT}\right)$ for a $3.18 \mu \mathrm{m}$ radius disk, $(\mathrm{j}, H)=\left(0.37 \times 10^{11} \mathrm{~A} / \mathrm{m}^{2}, 4 m T\right)$ for a $1.07 \mu \mathrm{m}$ radius disk, and $(\mathrm{j}, H)=\left(0.67 \times 10^{11} \mathrm{~A} / \mathrm{m}^{2}, 11 \mathrm{mT}\right)$ for a $0.54 \mu \mathrm{m}$ radius disk. (c) Electrically detected resonance frequency as a function of the disk radius in comparison to calculations based on equation (2).
We used spin-polarized alternating currents to excite the gyrotropic mode of the vortex core in disks with lateral electrical contacts. The dynamic response of the vortex core in the disk was measured electrically using the setup shown in Figure 2(a) and described in detail in the Methods section. The resonance frequencies measured on disks with different radii but constant Py thickness (25 $\mathrm{nm}$ ) are shown in Figure 2(b). In accordance with theory, the resonance frequency is higher for disks with smaller radii. When the equilibrium position of the core is shifted with an external field, the resistance change due to AMR will be different for the core displaced either to the right or left, enabling the detection of a rectified DC voltage. The gyration of the vortex core causes an oscillating change in resistance which allows for the detection of the resonance frequency. We found that the experimentally measured resonance frequencies are in good agreement with calculations based on the modified Thiele equation ${ }^{22}$, as summarised in Figure 2(c).

The measurement technique involves shifting of the equilibrium position of the vortex core into regions of different $M_{\mathrm{s}}$ by applying a static in-plane field. Therefore, we have first carefully studied the influence of the in-plane field on the resonance frequency for non-irradiated devices. The results of the measurements on a non-irradiated disk with design dimensions $3 \mu \mathrm{m}$ radius ( $30 \mathrm{~nm}$ Py) for a current density of $0.25 \times 10^{11} \mathrm{~A} / \mathrm{m}^{2}$ are shown in Supplementary Figure S1; the resonance frequency was determined to be $34 \mathrm{MHz}$. This confirms the theoretical expectations that there is no significant shift in the resonance frequency with the applied in-plane field, when the field is kept well-below the vortex annihilation field ${ }^{25}$. It is worth mentioning that, as expected, no signal could be detected close to $0 \mathrm{mT}$, due to symmetry arguments ${ }^{26}$.

The effects of ion implantation in ferromagnetic films with $3 d$ non-ferromagnetic metals have been intensively studied. Ion irradiation in case of Py typically yields a reduction of the Curie temperature and a reduction of saturation magnetization at room temperature. ${ }^{27-30}$. To introduce areas of different saturation magnetization in pre-fabricated Py disks, we used local $\mathrm{Cr}^{+}$implantation at an acceleration energy of $E_{C r}=30 \mathrm{keV}$ and an ion fluence of 1.2 $\times 10^{16}$ ions $/ \mathrm{cm}^{2}$. These parameters were chosen since they were identified as yielding the largest reduction of the saturation magnetisation of $\mathrm{Py}$, according to our initial characterisation results from magnetometry on irradiated continuous Py films. Chromium implantation was carried out with a broad beam source implanter, making it necessary to mask the regions of the disk where ion irradiation is not desired by positive photoresist. For this reason, a concentric disk or ring is exposed using electron beam lithography within the already existing disk, yielding a corresponding region of reduced $M_{\mathrm{s}}$ after irradiation. This, consequently, generates a complex magnetostatic potential within the disk. When the sample is irradiated in the middle, for example, the resulting effective magnetostatic potential is the convolution of two parabola-like shaped potentials. This change in the potential, compared to a single isolated non-irradiated disk, will also change its derivative, resulting in different frequencies when the core is placed in the different $M_{\mathrm{s}}$ regions.

Figure 3(a) shows a scanning electron microscopy (SEM) image (taken before patterning the contact pads) of a $35 \mathrm{~nm}$ 
thick, $3.18 \mu \mathrm{m}$ radius Py disk where $\mathrm{Cr}^{+}$was implanted into the outer ring. A clear contrast can be observed between the irradiated (dark) and non-irradiated (bright) region. Energy dispersive X-ray spectroscopy (EDXS) analysis confirms that $\mathrm{Cr}^{+}$is uniformly implanted only within the areas not protected by photoresist, as seen in supplementary information Figure S5(b). The vortex core can be positioned in either of the two different magnetisation regions by applying an external in-plane field, as done here, or, alternatively, by adjusting the current density. The electrically measured resonance frequencies are given in Figure 3(b), as measured at a
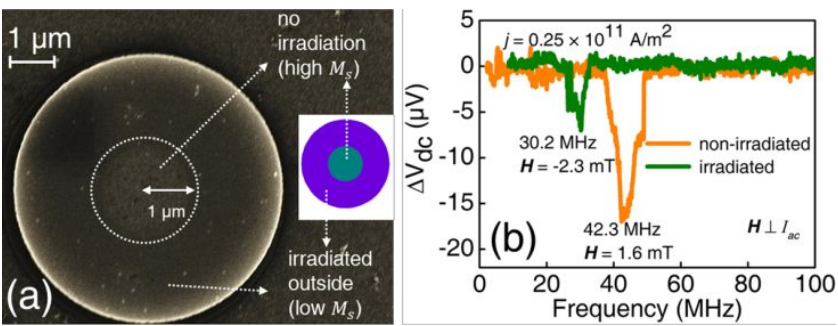

Figure 3: (a) SEM image of a $3.18 \mu \mathrm{m}$ radius disk implanted with chromium ions in the outer region with a ring width of $2 \mu \mathrm{m}$ (b) Modified resonance frequency measured with a current density of $0.20 \times 10^{11} \mathrm{~A} / \mathrm{m}^{2}$, an external magnetic field is used to shift the vortex between the two $M_{s}$ regions. The spectra were measured with applied fields of $-2.3 \mathrm{mT}$ and $1.6 \mathrm{mT}$ for the irradiated and non-irradiated regions, respectively.

current density of $0.20 \times 10^{11} \mathrm{~A} / \mathrm{m}^{2}$, for different vortex positions controlled with the external magnetic field. As expected, the device exhibits two discrete frequencies, 30.2 $\mathrm{MHz}$ and $42.3 \mathrm{MHz}$, corresponding to the vortex core precessing in the two different $M_{\mathrm{s}}$ regions, namely the outer, lower magnetisation $\operatorname{ring}\left(\mu_{0} M_{\mathrm{s}}=0.54 \mathrm{~T}\right)$, or the higher magnetisation $\left(\mu_{0} M_{\mathrm{s}}=0.92 \mathrm{~T}\right)$, non-irradiated inner region, respectively.

Spectra measured at different external fields corresponding to different vortex core positions in the device are shown in Figure 4. The disk was first saturated at $H=50 \mathrm{mT}$ before decreasing the field strength in order to allow for the vortex state to nucleate, starting with the core being located in the irradiated region at the rim. This yields a dip at approximately $30.2 \mathrm{MHz}$, first visible on the spectrum measured at $2.3 \mathrm{mT}$ (shown in green), corresponding to vortex precession in the irradiated area with reduced $\mu_{0} M_{\mathrm{s}}$. When sweeping the field further towards positive values, a small peak-like feature is observed at $0.7 \mathrm{mT}$ close to $42.3 \mathrm{MHz}$, showing that the vortex is now precessing in the unirradiated disk center. Further increasing the external field yields a spectrum exhibiting a clear dip - again at the same frequency (42.3 MHz) at $1.6 \mathrm{mT}$, as shown in orange. This sign change in the signal variation between peak-like and dip-

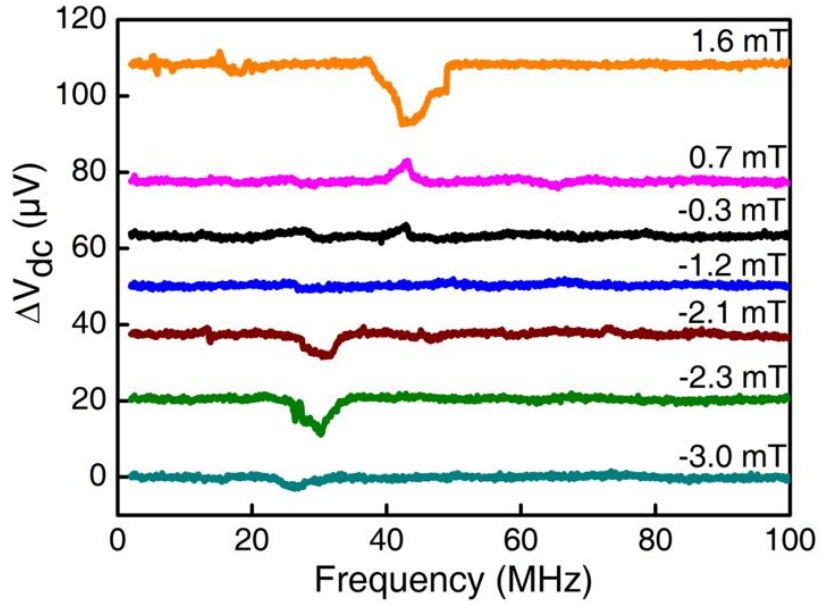

Figure 4: Obtained resonance frequencies from the disk shown in Figure 3(a) measured at a current density of $0.20 \times 10^{11} \mathrm{~A} / \mathrm{m}^{2}$ at different applied magnetic fields in the film plane in order to displace the vortex core (curves shifted vertically for clarity).

like is likely caused by the vortex core flipping due to continuous supply of RF current along with an external field $^{31}$. Above $1.6 \mathrm{mT}$, no response was detected, although it is expected that the vortex should continue shifting away from the center area and back in the irradiated region again. It is possible that the vortex core moved under the contacts (as field was applied perpendicular to current), in which case no signal can be detected, as this area is not probed by the flowing current.
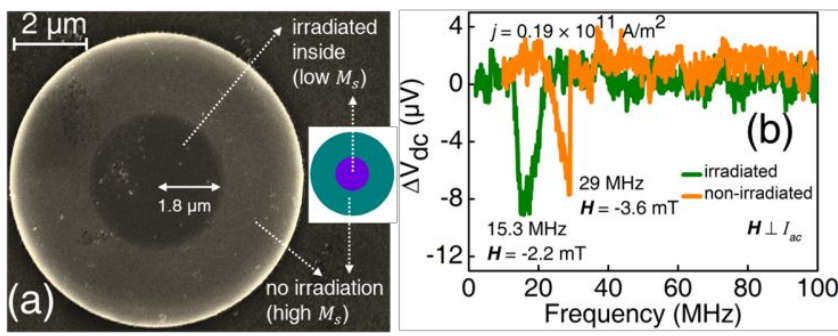

Figure 5: (a) SEM image of a $4.16 \mu \mathrm{m}$ radius disk (35 $\mathrm{nm}$ Py) implanted with chromium in the central region as a concentric disk with a radius of $1.8 \mu \mathrm{m}$ (b) Modified resonance frequency measured with a current density of $0.15 \times 10^{11} \mathrm{~A} / \mathrm{m}^{2}$, an external magnetic field is used to shift the vortex between the two regions.

Figure 5(a) shows an SEM image of a disk implanted in the center area. In this case, a concentric disk with $1.8 \mu \mathrm{m}$ radius was implanted with $\mathrm{Cr}^{+}$into a $4.16 \mu \mathrm{m}$ radius Py disk with a thickness of $35 \mathrm{~nm}$. The electrically measured resonance frequencies are given in Figure 5(b), as measured at a current density of $0.15 \times 10^{11} \mathrm{~A} / \mathrm{m}^{2}$. The measurements were conducted in a similar fashion as in the previous case. The sample was first saturated at a magnetic field of $H=50 \mathrm{mT}$, which is much higher than the annihilation field of the particular vortex structure. Decreasing the magnetic field first leads to a resonant frequency determined to be $29 \mathrm{MHz}$, corresponding to the non-irradiated pure Py in the outer disk region. While decreasing the magnetic field strength further, the vortex core displacement is reduced and another resonance is detected at around $15.3 \mathrm{MHz}$, this time originating from the 
central disk region with reduced $M_{\mathrm{s}}$ due to $\mathrm{Cr}^{+}$ implantation.

Table 1: Summary of average frequencies obtained from experiment and frequencies from micromagnetic simulation.

\begin{tabular}{|c|c|c|}
\hline Frequency Region & irradiated & non-irradiated \\
\hline electrically detected & $34.35 \mathrm{MHz}$ & $47.10 \mathrm{MHz}$ \\
\hline from simulation & $39.81 \mathrm{MHz}$ & $51.15 \mathrm{MHz}$ \\
\hline \multicolumn{2}{|c|}{$3.18 \mu \mathrm{m}$ radius, $35 \mathrm{~nm}$ Py disk irradiated outside } \\
\hline
\end{tabular}

\begin{tabular}{|c|c|c|}
\hline Frequency & irradiated & non-irradiated \\
\hline electrically detected & $18.18 \mathrm{MHz}$ & $25.72 \mathrm{MHz}$ \\
\hline from simulation & $22.19 \mathrm{MHz}$ & $29.31 \mathrm{MHz}$ \\
\hline
\end{tabular}

Experimentally detected frequencies averaged over several field values for both irradiated and non-irradiated regions (as mentioned in supporting information F), and values obtained from micromagnetic simulations are given in Table 1. The simulations confirm the reduction in $M_{\mathrm{s}}$ upon irradiation as the underlying mechanism of the independent gyrotropic frequencies.

It can be seen that the electrically measured frequencies are in good qualitative agreement with the simulated values. The order of magnitude of the discrepancy between measured and simulated values is about $10-20 \%$ and similar to the results obtained on disks with uniform magnetisation presented in Figure 2. Possible reasons for this discrepancy include a slightly imperfect disk shape in the experiment compared to the simulation, as well as subtle differences in the parameters assumed in the simulations and the real values (in particular the exchange constant). For the implanted disks, a small variation in height does exist between the irradiated and non-irradiated regions, $\sim 2.1 \mathrm{~nm}$ (see Supplementary Information, Fig. S4 and the relevant text), which is not taken into account in the simulations. Additionally, our choice of photoresist mask introduces a small shallow-implanted area at the border between the irradiated and non-irradiated regions, which the simulations also do not take into account (see Supplementary Information, Fig. S6 and the relevant text).

Conclusion: This work was aimed at realizing single magnetic vortex devices that output multiple, discrete frequencies upon resonant excitation. We have explored the possibility to detect the resonance frequencies associated with the different areas of the chromium implanted disks with radii of $3.18 \mu \mathrm{m}$ and $4.16 \mu \mathrm{m}$. Concentric regions of single disks were implanted with chromium ions and the modified dynamics were electrically measured. Two distinct frequencies were successfully detected electrically from single Py disks implanted with $\mathrm{Cr}$. Achieving different resonance frequencies within a single physical disk shows that multiple working frequencies can be exploited without further lithographical steps. Given that ion irradiation with sufficiently large fluence $\left(1.5 \times 10^{16} \mathrm{ions} / \mathrm{cm}^{2}\right)$ can be used to transform a ferromagnetic material into a paramagnet ${ }^{30}$, a similar approach could be used in order to magnetically pattern several magnetic vortices into a non-magnetic or weakly ferromagnetic matrix, allowing for the vortices to be spaced within distances that are unattainable by conventional patterning techniques. This approach has thus the potential to design closely-packed networks of oscillators, while at the same time improving the coupling between neighboring devices. Indeed, in order to show that this principle applies to current driven nano-oscillators, we have simulated a permalloy disk with $500 \mathrm{~nm}$ diameter with an inner region with a diameter of $100 \mathrm{~nm}$ and $60 \%$ of the permalloy saturation magnetisation. The gyrotropic precession of the vortex core was excited with spinpolarised current flowing out-of-plane. As shown in the Supplementary Movie, for a given current density, the vortex core gyrates with a given frequency $390 \mathrm{MHz}$ in the inner region. By increasing the current density, the vortex core moves to the outer region with smaller saturation magnetisation and thus resulting in a gyration with a lower frequency of $245 \mathrm{MHz}$. Simulations also show that the vortex core can be trapped in the region between the different saturation magnetisations and thus the dynamics is suppressed. Note that direct irradiation at sub-100 nm scale is nowadays possible with modern ion irradiation technology and could, in principle, enable the creation of vortex nano-oscillators capable of functioning at multiple frequencies, beyond the limit of what can be achieved with conventional patterning techniques ${ }^{32}$.

Methods: Permalloy (Py- $\mathrm{Ni}_{80} \mathrm{Fe}_{20}$ ) disks with different radii and thickness are prepared by means of electron beam lithography, material deposition using electron beam evaporation followed by conventional lift-off technique. Each disk is contacted individually by $125 \mathrm{~nm}$ thick $\mathrm{Au}$ electrodes to allow for electrical connection. $5 \mathrm{~nm} \mathrm{Cr}$ is used in both cases for better adhesion to the Si substrate. Three sets of samples are discussed in this work: The first set of samples has individual Py disks without any electrical contacts for preliminary study (cf. Figure 1). The second set of samples consists of individual Py disks with electrical contacts (cf. Figure 2) on which electrical detection of the magnetic vortices was performed. The third set of samples also contain individual Py disks but is implanted with chromium (cf. Figures 3-5) to investigate the modified magnetic properties upon implantation as described in the article. The contacts are deposited and patterned after the chromium implantation.

The presence of vortex states in the patterned disks was verified by means of X-ray photoemission electron microscopy (XPEEM). The XPEEM end station at the Berlin Electron Storage Ring Society for Synchrotron Radiation (BESSY II) was used to image the magnetic domains of inplane magnetisation in the magnetic vortex structures. XPEEM uses the local variations in electron emission to generate an image $^{33}$ which can be used to map the magnetisation configurations in nanoscale structures ${ }^{34}$. The spin-polarised PEEM setup at BESSY II is attached to an undulator beamline with full polarization control (UE49) and has a variable field of view ( $3 \mu \mathrm{m}$ to $70 \mu \mathrm{m})$. It is mostly sensitive to the in-plane magnetisation component as the incident angle of X-rays is fixed perpendicular to the sample surface at an angle of $74^{\circ}$. The sample is exposed to X-rays generated by an undulator and due to varied beam energy, photoelectrons can be resonantly excited. The secondary electrons generated are directly proportional to the energy dependent X-ray absorption of the sample material. The magnetic contrast is provided by X-ray magnetic circular dichroism (XMCD) that detects the difference in X-ray 
absorption of circularly polarized X-rays (measured at material specific absorption edges) depending on the helicity (left handed vs. right handed) of the photon beam and the magnetisation at the sample surface ${ }^{35}$. The magnetic contrast is calculated as the difference of two PEEM images recorded with opposite helicities divided by their sum and represents a projection of the sample magnetisation on the incident X-ray beam.

Dynamic measurements: The dynamic response of the vortex core in the disk is measured electrically. The injected microwave current with frequency $\omega, I_{a c}=I_{0} \cos \omega t$ gives rise to a resistance oscillation $\Delta R=\Delta R_{0} \cos (\omega t+\varphi)$, with $\varphi$ being the phase shift between the resistance change and the injected current. This leads to a voltage with one frequencydependent and one frequency-independent term:

$$
V=\frac{I_{0} \Delta R_{0}}{2} \cos (2 \omega t+\varphi)+\frac{I_{0} \Delta R_{0}}{2} \cos (\varphi)
$$

The frequency-independent term is the rectified DC voltage which is measured by the lock-in amplifier,

$$
V_{d c}=\frac{I_{0} \Delta R_{0}}{2} \cos (\varphi)
$$

This equation is based on the assumption that the resistance oscillations are at the same frequency as the injection current ${ }^{36}$, which is not the case here as the AMR oscillations are produced at twice the frequency, $2 \omega$. Due to the frequency mismatch between the injected microwave current and the AMR oscillation, the microwave current cannot be rectified. Previous works also demonstrate that it is possible to probe the $2 \omega$ signal $^{37,38}$. However, by displacing the vortex with a small in-plane static field, the $V_{d c}$ can be measured ${ }^{26}$ as presented here. To improve the signal-to-noise ratio, lock-in detection was adopted with the microwave current being amplitude modulated at a frequency of $223 \mathrm{~Hz}$. All the results shown in this work correspond to the external magnetic field applied perpendicular (in the same plane) to the current measurement direction unless specified otherwise.

Micromagnetic simulations: Micromagnetic simulations were performed by numerically solving the Landau-Lifshitz-Gilbert equation of motion using the GPU accelerated code $\operatorname{MuMax}_{3}{ }^{39}$. For all simulations the disks with Permalloy material parameters, $\mu_{0} M_{\mathrm{s}}=0.92 \mathrm{~T}(0.54 \mathrm{~T}$ for the irradiated region), $A=13 \mathrm{pJ} / \mathrm{m}$, was discretized into cell smaller that the exchange length. The Gilbert damping parameter was set to 0.008 .

Similar to the experimental scenario, the vortex core was displaced with an in-plane field to the regions of different saturation magnetization. The gyrotropic precession around the equilibrium position was then excited with a small in-plane field pulse. Due to the large radius of the disk ( $8 \mu \mathrm{m}$ in diameter) the gyration frequencies are small. Therefore, the magnetisation dynamics was recorded over several hundreds of nanoseconds to determine the gyration frequency.

\section{ASSOCIATED CONTENT}

Supporting Information.

Additional information including results from magnetometry, electrical measurements and simulations (PDF)

Movie from micromagnetic simulations showing the gyrotropic precession of the vortex core in one disk with varying saturation magnetisation (MPG)
This material is available free of charge via the Internet at http://pubs.acs.org.

\section{AUTHOR INFORMATION}

\section{Corresponding Author}

*l.ramasubramanian@hzdr.de.

\section{Author Contributions}

These authors contributed equally. L.R., A.K., C.F and A.D. designed the experiments. A.K. and O.Y. performed the $\mathrm{MuMax}_{3}$ and TRIM simulations, respectively. L.R. prepared the samples and performed the electrical measurements. S.S. contributed to the discussions in electrical measurements. P.M. carried out the MOKE measurements. A.T. did the magnetometry and D.H. the MFM measurements. R.B. conducted ion-irradiations. F.K. and D.M. executed the XMCD measurements. R.H. conducted the TEM and EDXS measurements.

L.R. and A.K. wrote the manuscript with input from all authors. All authors have given approval to the final version of the manuscript.

\section{ACKNOWLEDGMENT}

This work is supported by the Helmholtz Young Investigator Initiative Grant No. VH-N6-1048. Support by the Nanofabrication Facilities Rossendorf at IBC is gratefully acknowledged. The authors acknowledge Dr. Shengqiang Zhou for providing SQUID measurement time and assisting with the measurements. The use of HZDR Ion Beam Center TEM facilities and the funding of TEM Talos by the German Federal Ministry of Education of Research (BMBF) under Grant No. 03SF0451 in the framework of HEMCP are acknowledged. We acknowledge financial support through the European Regional Development Fund (EFRE) and the Free State of Saxony, as well as the Fraunhofer Internal Programmes under Grant No. MEF 836303. This work was financed in part via the German Research Foundation (DFG) Grants No. MA5144/9-1, MA5144/14-1, No. MA5144/22-1. We thank HZB for the allocation of synchrotron radiation beamtime.

\section{ABBREVIATIONS}

AMR, Anisotropic magnetoresistance; Py, Permalloy; XMCDPEEM, X-ray magnetic circular dichroism- Photoemission electron microscopy; SEM, Scanning electron microscopy.

\section{REFERENCES}

(1) Deac, A. M.; Fukushima, A.; Kubota, H.; Maehara, H.; Suzuki, Y.; Yuasa, S.; Nagamine, Y.; Tsunekawa, K.; Djayaprawira, D. D.; Watanabe, N. Bias-Driven High-Power Microwave Emission from MgO-Based Tunnel Magnetoresistance Devices. Nat. Phys. 2008, 4, 803-809.

(2) Lee, K.-J.; Deac, A.; Redon, O.; Nozières, J.-P.; Dieny, B. Excitations of Incoherent Spin-Waves Due to Spin-Transfer Torque. Nat. Mater. 2004, 3, 877-881.

(3) Maehara, H.; Kubota, H.; Suzuki, Y.; Seki, T.; Nishimura, K.; Nagamine, Y.; Tsunekawa, K.; Fukushima, A.; Deac, A. M.; Ando, K.; Yuasa, S. Large Emission Power over 2 MW with High Q Factor Obtained from Nanocontact Magnetic-Tunnel-Junction-Based Spin Torque Oscillator. Appl. Phys. Express 2013, 6, 113005.

(4) Dussaux, A.; Georges, B.; Grollier, J.; Cros, V.; Khvalkovskiy, A. V.; Fukushima, A.; Konoto, M.; Kubota, H.; Yakushiji, K.; Yuasa, S.; Zvezdin, K. A.; Ando, K.; Fert, A. Large Microwave Generation from Current-Driven Magnetic Vortex Oscillators in Magnetic Tunnel Junctions. Nat. Commun. 2010, 1, 8. (5) Pribiag, V. S.; Krivorotov, I. N.; Fuchs, G. D.; Braganca, P. M.; Ozatay, O.; Sankey, J. C.; Ralph, D. C.; Buhrman, R. A. Magnetic 
Vortex Oscillator Driven by d.c. Spin-Polarized Current. Nat. Phys., 2007, 3, 498-503.

(6) Mistral, Q.; van Kampen, M.; Hrkac, G.; Kim, J.-V.; Devolder, T.; Crozat, P.; Chappert, C.; Lagae, L.; Schrefl, T. CurrentDriven Vortex Oscillations in Metallic Nanocontacts. Phys. Rev. Lett., 2008, 100, 257201.

(7) Suess, D.; Bachleitner-Hofmann, A.; Satz, A.; Weitensfelder, H.; Vogler, C.; Bruckner, F.; Abert, C.; Prügl, K.; Zimmer, J.; Huber, C.; Luber, S.; Raberg, W.; Schrefl, T.; Brückl, H. Topologically Protected Vortex Structures for Low-Noise Magnetic Sensors with High Linear Range. Nat. Electron. 2018, 1, 362-370.

(8) Rippard, W. H.; Pufall, M. R.; Kaka, S.; Russek, S. E.; Silva, T. J. Direct-Current Induced Dynamics in $\mathrm{Co}_{90} \mathrm{Fe}_{10} / \mathrm{Ni}_{80} \mathrm{Fe}_{20}$ Point Contacts. Phys. Rev. Lett. 2004, 92 (2), 90-93.

(9) Jenkins, A. S.; San Emeterio Alvarez, L.; Dutra, R.; Sommer, R. L.; Freitas, P. P.; Ferreira, R. A multi-band analogue frequency sensor with sub-MHz resolution based on a Vortex Nano-Oscillator. arXiv:1806.06002

(10) Behncke, C.; Adolff, C. F.; Wintz, S.; Hänze, M.; Schulte, B.; Weigand, M.; Finizio, S.; Raabe, J.; Meier, G. Tunable Geometrical Frustration in Magnonic Vortex Crystals. Sci. Rep. 2018, 8, 186.

(11) Romera, M.; Talatchian, P.; Tsunegi, S.; Araujo, F. A.; Cros, V.; Bortolotti, P.; Trastoy, J.; Yakushiji, K.; Fukushima, A.; Kubota, H.; Yuasa, S.; Ernoult, M.; Vodenicarevic, D.; Hirtzlin, T.; Locatelli, N.; Querlioz, D.; Grollier, J. Vowel Recognition with Four Coupled Spin-Torque Nano-Oscillators. Nature 2018, 563, 230-234.

(12) Silver, D.; Schrittwieser, J.; Simonyan, K.; Antonoglou, I.; Huang, A.; Guez, A.; Hubert, T.; Baker, L.; Lai, M.; Bolton, A.; Chen, Y.; Lillicrap, T.; Hui, F.; Sifre, L.; van den Driessche, G.; Graepel, T.; Hassabis, D. Mastering the Game of Go without Human Knowledge. Nature 2017, 550, 354-359.

(13) Sussillo, D. Neural Circuits as Computational Dynamical Systems. Curr. Opin. Neurobiol. 2014, 25, 156-163.

(14) Borisyuk, R.; Denham, M.; Hoppensteadt, F.; Kazanovich, Y.; Vinogradova, O. An Oscillatory Neural Network Model of Sparse Distributed Memory and Novelty Detection. In BioSystems 2000, 58, 265-272.

(15) Fang, Y.; Yashin, V. V.; Levitan, S. P.; Balazs, A. C. Pattern Recognition with "Materials That Compute." Sci. Adv. 2016, 2(9), $1-10$.

(16) Torrejon, J.; Riou, M.; Araujo, F. A.; Tsunegi, S.; Khalsa, G.; Querlioz, D.; Bortolotti, P.; Cros, V.; Yakushiji, K.; Fukushima, A.; Kubota, H.; Yuasa, S.; Stiles, M. D.; Grollier, J. Neuromorphic Computing with Nanoscale Spintronic Oscillators. Nature 2017, 547, 428-431.

(17) Wachowiak, A.; Wiebe, J.; Bode, M.; Pietzsch, 0.; Morgenstern, M.; Wiesendanger, R. Direct Observation of Internal Spin Structure of Magnetic Vortex Cores. Science, 2002, 298, 577580.

(18) Vavassori, P.; Grimsditch, M.; Metlushko, V.; Zaluzec, N.; Ilic, B. Magnetoresistance of single magnetic vortices. Appl. Phys. Lett., 2005, 86, 072507.

(19) Kikuchi, N.; Okamoto, S.; Kitakami, O.; Shimada, Y.; Kim, S. G.; Otani, Y.; Fukamichi, K. Vertical Bistable Switching of Spin Vortex in a Circular Magnetic Dot. J. Appl. Phys. 2001, 90, 6548.

(20) Kasai, S.; Nakatani, Y.; Kobayashi, K.; Kohno, H.; Ono, T. Current-Driven Resonant Excitation of Magnetic Vortices. Phys. Rev. Lett. 2006, 97, 107204.

(21) Thiele, A. A. Steady-State Motion of Magnetic Domains. Phys. Rev. Lett. 1973, 30, 230-233.

(22) Guslienko, K. Yu.; Ivanov, B. A.; Novosad, V.; Otani, Y.; Shima, H.; Fukamichi, K. Eigenfrequencies of Vortex State
Excitations in Magnetic Submicron-Size Disks. J. Appl. Phys. 2002, 91, 8037.

(23) Ding, J.; Lapa, P.; Jain, S.; Khaire, T.; Lendinez, S.; Zhang, W.; Jungfleisch, M. B.; Posada, C. M.; Yefremenko, V. G.; Pearson, J. E.; Hoffmann, A.; Novosad, V.. Spin Vortex Resonance in Non-Planar Ferromagnetic Dots. Sci. Rep. 2016, 6, 25196.

(24) Guslienko, K. Yu.; Novosad, V.; Otani, Y.; Shima, H.; Fukamichi, K. Magnetization Reversal Due to Vortex Nucleation, Displacement, and Annihilation in Submicron Ferromagnetic Dot Arrays. Phys. Rev. B 2002, 65, 024414.

(25) Aliev, F. G.; Sierra, J. F.; Awad, A. A.; Kakazei, G. N.; Han, D. -S.; Kim, S. -K.; Metlushko, V.; Ilic, B.; Guslienko, K. Yu. Spin Waves in Circular Soft Magnetic Dots at the Crossover between Vortex and Single Domain State. Phys. Rev. B 2009, 79, 174433.

(26) Sushruth, M.; Fried, J. P.; Anane, A.; Xavier, S.; Deranlot, C.; Kostylev, M.; Cros, V.; Metaxas, P. J. Electrical Measurement of Magnetic-Field-Impeded Polarity Switching of a Ferromagnetic Vortex Core. Phys. Rev. B 2016, 94, 100402(R).

(27) Gupta, R.; Lieb, K. P.; Luo, Y.; Müller, G. A.; Schaaf, P.; Zhang, K. Argon and Krypton Ion-Induced Changes in Permalloy Thin Films. Eur. Phys. J. B 2008, 63, 501-506.

(28) Folks, L.; Fontana, R. E.; Gurney, B. A.; Childress, J. R.; Maat, S.; Katine, J. A.; Baglin, J. E. E.; Kellock, A. J. Localized Magnetic Modification of Permalloy Using $\mathrm{Cr}^{+}$Ion Implantation. J. Phys. D. Appl. Phys. 2003, 36, 21, 2601-2604.

(29) Fassbender, J.; McCord, J. Control of Saturation Magnetization, Anisotropy, and Damping Due to Ni Implantation in Thin $\mathrm{Ni}_{81} \mathrm{Fe}_{19}$ layers. Appl. Phys. Lett. 2006, 88, 252501.

(30) Fassbender, J.; Von Borany, J.; Mücklich, A.; Potzger, K.; Möller, W.; McCord, J.; Schultz, L.; Mattheis, R. Structural and Magnetic Modifications of Cr-Implanted Permalloy. Phys. Rev. B 2006, 73, 184410.

(31) Van Waeyenberge, B.; Puzic, A.; Stoll, H.; Chou, K. W.; Tyliszczak, T.; Hertel, R.; Fähnle, M.; Brückl, H.; Rott, K.; Reiss, G.; Neudecker, I.; Weiss, D.; Back, C. H.; Schütz, G. Magnetic Vortex Core Reversal by Excitation with Short Bursts of an Alternating Field. Nature 2006, 444, 461-464.

(32) Nord, M.; Semisalova, A.; Kákay, A.; Hlawacek, G.; MacLaren, I.; Liersch, V.; Volkov, O. M.; Makarov, D.; Paterson, G. W.; Potzger, K.; Lindner, J.; Fassbender, J.; McGrouther, D.; Bali, R. Strain Anisotropy and Magnetic Domains in Embedded Nanomagnets. Small 2019, 15, 1904738.

(33) Brundle, C. R.; Baker, A. D. Electron Spectroscopy: Theory, Techniques and Applications 1979, 3 (Academic Press).

(34) J. Stöhr; H.C. Siegmann. Magnetism: From fundamentals to Nanoscale Dynamics (Springer, 2006).

(35) Streubel, R.; Fischer, P.; Kronast, F.; Kravchuk, V. P.; Sheka, D. D.; Gaididei, Y.; Schmidt, O. G.; Makarov, D. Magnetism in Curved Geometries. J. Phys. D: Appl. Phys. 2016, 49, 363001.

(36) Kim, J. -S.; Boulle, O.; Verstoep, S.; Heyne, L.; Rhensius, J.; Kläui, M.; Heyderman, L. J.; Kronast, F.; Mattheis, R.; Ulysse, C.; Faini, G. Current-Induced Vortex Dynamics and Pinning Potentials Probed by Homodyne Detection. Phys. Rev. B 2010, 82, 104427.

(37) Sugimoto, S.; Hasegawa, N.; Niimi, Y.; Fukuma, Y.; Kasai, S.; Otani, Y. Detection of a Symmetric Circular Gyration of the Vortex Core via the Second-Order Harmonic Magnetoresistance Oscillation. Appl. Phys. Express 2014, 7, 023006.

(38) Cui, X.; Yakata, S.; Kimura, T. Detection of Vortex Core Oscillation Using Second-Harmonic Voltage Detection Technique. IEEE Trans. Magn. 2015, 51, 4300303.

(39) Vansteenkiste, A.; Leliaert, J.; Dvornik, M.; GarciaSanchez, F.; Van Waeyenberge, B. The Design and Verification of Mumax ${ }^{3}$. AIP Advances 2014, 4, 107133. 


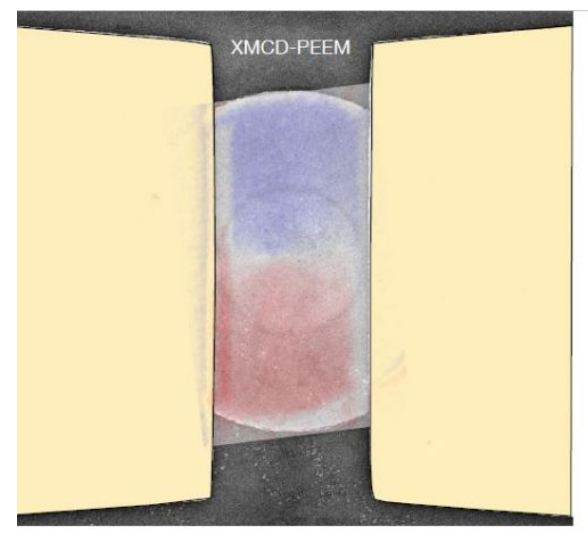

$\mathrm{H}_{\text {ext }}=0 \mathrm{mT}$

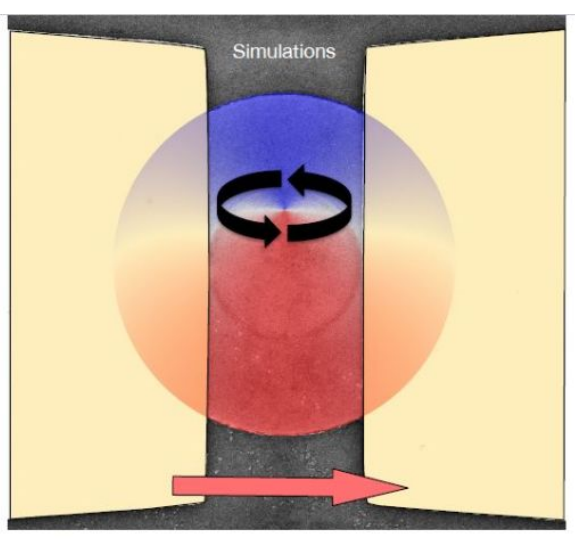

$H_{\text {ext }}=-2.2 m T$

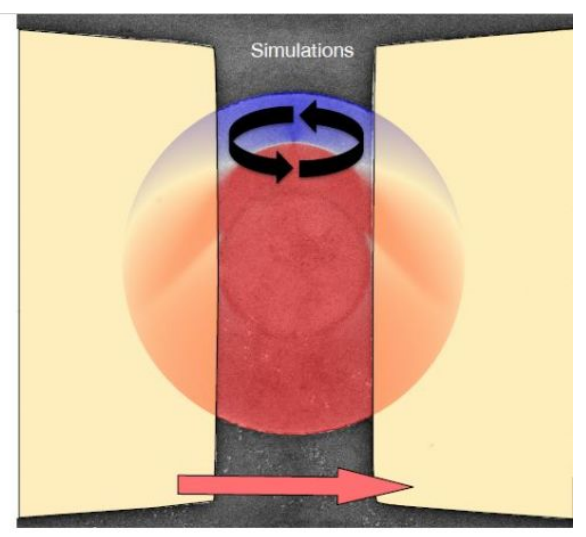

$H_{\text {ext }}=-3.6 m T$ 